\title{
La integración en Latinoamérica: entre el regionalismo cerrado y el regionalismo abierto
}

\section{Integration in Latin America: between closed regionalism and open regionalism}

Manuel Antonio Mejía Baños ${ }^{1}$, Moisés Edgardo Meriño Polo ${ }^{2}$, Clara Patricia Ruiz Araque ${ }^{3}$

INFORMACIÓN DEL

\section{ARTÍCULO}

Fecha de recepción: 1 de Septiembre de 2020.

Fecha de aceptación: 10 de Noviembre de 2020 .

${ }^{1}$ Magister en In Scienze Politiche Per La Pace El Integrazione Dei Popoli, Università Degli Studi di Salerno di Italia. Docente-investigador, Fundación Universitaria los Libertadores-Colombia.

E-mail: mamejiab@libertadores.edu.co Código ORCID:

https://orcid.org/0000-0001-7952-0719

2 Magíster en Auditoría Internacional, Universidad Internacional Iberoamérica. Docente-investigador, Fundación Universitaria los Libertadores-Colombia.

E-mail:

moises.merino@libertadores.edu.co

Código ORCID:

https://orcid.org/0000-0001-8184-8211

3 Economista, Universidad Central de Colombia. Docente-investigadora, Universidad Distrital Francisco José de CaldasColombia.

E-mail: cpruiza@udistrital.edu.co

Código ORCID:

https://orcid.org/0000-0002-6103-6619

CITACIÓN: Mejía Baños, M.A. Meriño Polo, M.E., \& Ruíz Araque, C.P. (2020). La integración en Latinoamérica: entre el regionalismo cerrado y el regionalismo abierto. Podium, 38, 105-122.

doi:10.31095/podium.2020.38.7

ENLACE DOI:

http://dx.doi.org/10.31095/podium.202 0.38 .7

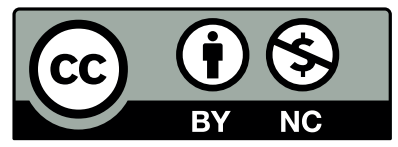

\section{Resumen}

El objetivo del presente artículo de investigación es identificar si el exceso de ideología en las iniciativas de integración materializadas en el regionalismo cerrado de la CAN, CELAC, ALBA, MERCOSUR y el regionalismo abierto de la Alianza del Pacífico, han limitado el alcance de objetivos sociales y económicos en los países de América Latina, para lo cual se utilizó una investigación cualitativa de orden descriptivo. Se concluyó que en cada una de las iniciativas estudiadas se observan fuertes componentes ideológicos, que dificultan establecer puntos de convergencias que permitan concurrir al alcance de objetivos sociales y económicos conjuntos, lo cual debilita la capacidad de la región para mostrarse al mundo como un bloque sólido que busque ser un actor determinante en la política y economía mundial.

\section{Palabras Clave:}

Integración, Desigualdad, Pobreza, Comercio, Mercados, Bienestar. Clasificación JEL: F020, F150.

\begin{abstract}
The present research paper purports to identify whether excess ideology in closed or open regionalism has limited social and economic objectives reach in Latin-American. Closed regionalism belongs to CAN, CELAC, ALBA, MERCOSUR; open regionalism belongs to Alianza del Pacífico. Research was qualitative and descriptive. Strong ideological components were observed in each of the initiatives that were studied. Ideological components made it difficult to establish convergence points which would permit reaching join social and economics objectives. The region's ability to appear as a solid block is weakened by lack of convergence points. This block would, otherwise, sick to be a determinating actor in world policy and economy. It was concluded that in each of the initiatives studied, a strong ideological component is observed, that makes it difficult to establish points of convergence that allow the achievement of joint social and economic objectives, which weakens the region's ability to show itself to the world as a solid block that attempts to be a determining factor in politics and world economy.
\end{abstract}

\section{Keywords:}

Integration, Inequality, Poverty, Trade, Market, Wellness.

JEL Classification: F020, F150.

$$
105
$$

PODIUM No. 38, Diciembre 2020, pp. 105-122

(C) Universidad Espíritu Santo - UEES

ISSN: 1390-5473 e-ISSN: 2588-0969 


\section{Introducción}

En Latinoamérica los procesos de integración, históricamente han presentado un importante dinamismo que para algunos son más retóricos que prácticos, no obstante, para Mejia (2018) "los procesos de integración en América Latina se pueden considerar endémicos, desde el nacimiento mismo de las repúblicas con el proyecto político de Bolívar y las propuestas culturales de Andrés Bello" (p. 217). En esa misma línea, Guadarrama $(2004 ; 2019)$ plantea que la integración en América Latina es evidente en el pensamiento político de Miranda, Bolívar y Martí. Sin embargo, desde comienzos del siglo XXI, se presentaron grandes cambios en esta materia, con la llegada al poder de gobernantes como los de: Venezuela con Hugo Rafael Chávez Frías, Bolivia con Evo Morales, Ecuador con Rafael Correa, Argentina con Cristina Fernández, Brasil con Luiz Inácio Lula da Silva, entre otros, quienes defienden $\mathrm{y}$ practican el socialismo del siglo XXI, en donde las mejoras en las condiciones de vida de la población juegan un papel relevante, por lo que impulsaron iniciativas de integración como el ALBA y UNASUR. Estas, con un desarrollo de carácter socialista, basadas en la planificación económica, donde los gobiernos $\mathrm{y}$ pueblos de Latinoamérica y el Caribe se organizan como una sola gran Nación para afrontar retos y desafíos generales en el ámbito económico, político, ambiental, social y cultural, rompiendo, de cierta manera, con el carácter hegemónico de Estados Unidos en términos económicos, políticos y culturales en la región. No obstante, existen países como Colombia, Perú, Chile y México, con tendencias políticas y económicas basadas en la integración hacia fuera, el libre mercado (desregulación económica y comercial) como estrategia de desarrollo, quienes pretenden profundizar su inserción económica con los países de Asia-Pacífico a través de la Alianza del Pacífico, propuesta que se contrapone de manera directa a la del ALBA y UNASUR.

En este sentido, el presente artículo analiza, de manera comparada, algunos de los procesos de integración vigentes como: la CAN, el ALBA, la CELAC y el MERCOSUR en América Latina, con la Alianza del Pacífico, con el propósito de identificar elementos coincidentes $y$ divergentes que permitan entender la pluralidad de acuerdos y la lucha ideológica entre ellos. Sin embargo, en este documento se aborda la integración desde tres perspectivas:

La hobbesiana o realista, que ve la política internacional como un estado de guerra; la tradición kantiana o universalista, que ve en el ejercicio de la política internacional una potencial comunidad humana; y la tradición grosciana o internacionalista que ve la política internacional como una realidad en el encuadramiento de la sociedad internacional (Bull, 1977, p.24).

En la postura Hobbesiana, se puede decir que los Estados se encuentran en conflicto permanente, y cada uno asigna su propio fin, oponiéndose así a la idea de integración para alcanzar objetivos comunes, la Kantiana, sigue el 
antecedente fundamental de la concepción integracionista, partiendo de la cooperación entre los Estados gracias a lazos transnacionales de unión $\mathrm{y}$ de acercamiento entre individuos de una comunidad humana con intereses similares, que podrían conducir incluso a la desaparición del sistema de Estados; y la Grosciana, es un punto medio entre los dos anteriores, de la cual no admite el conflicto generalizado Hobbesiano y tampoco el optimismo Kantiano de interés generalizado entre los Estados. Esta postura está basada en una sociedad de estados fundada en reglas institucionales fuertes, donde convergen todos los asociados y se estimula el intercambio comercial bajo las premisas de respeto y confianza (Vieira, 2005).

\section{Revisión de la literatura}

Haas (1958) define la integración política como "el proceso por el que los actores políticos en varios marcos nacionales distintos se persuaden a cambiar sus lealtades, expectativas y actividades políticas hacia un nuevo centro, cuyas instituciones poseen $\mathrm{o}$ piden jurisdicción sobre los Estados nacionales preexistentes", (p.1252). Postura cercana a los criterios de organizaciones supranacionales neofuncionalistas.

De igual manera, otros autores clasifican las organizaciones internacionales, resultantes de los procesos de integración, en supranacionales $\mathrm{o}$ intergubernamentales. "Las supranacionales según cuentan con poder decisorio que vincula a los Estados sin su consentimiento y con efecto directo sobre sus nacionales" (Tremolada, 2015, p.743). Mientras tanto, las organizaciones intergubernamentales demandan consentimiento de los Estados para vincularlos (Urueña, 2008).

Penneta (2011) por su parte, considera que la integración en América Latina no es más que retórica y se encuentra estancada por la falta de voluntad política de los gobiernos, quienes se caracterizan por establecer declaraciones ostentosas, pero sin iniciativas prácticas. Por otro lado, Caballero (2013) establece que los diferentes proyectos de integración regional rivalizan, se solapan y generan sinergias en América Latina. En este sentido, están en competición dos lógicas de inserción internacional. Para Márquez y Recalde (2017), existe un gran solapamiento de funciones y poderes entre los países de la región, debido a la existencia simultánea de por menos ocho acuerdos, lo cual limita una correcta coordinación en materia de integración económica en la región que permita alcanzar objetivos comunes. En ese sentido, Botero (2013) establece que se percibe cierta vanidad en los protagonistas de dichos procesos; al parecer, todos quieren liderar la integración, haciendo que los intereses y objetivos de sus naciones sobresalgan más allá de los demás. Por lo tanto, Botto (2015) plantea que la historia de América Latina, en materia de integración regional, es larga y errática, debido a que existen múltiples iniciativas, pero sin resultados contundentes.

Grynspan (2015) plantea que América 
Latina debe desarrollar una nueva generación de políticas públicas y acuerdos nacionales e intrarregionales en plazos que vayan más allá de los períodos gubernamentales, con una visión a largo plazo, lo cual redundaría en procesos de integración más sostenibles, dinámicos e incluyentes; en este sentido, Aranda y Salinas (2015) establecen que el ALBA y Alianza del Pacífico son parte de dos esquemas de cooperación con diferencias ideológicas, pero también con elementos comunes, el ALBA desea promover el comercio justo, eliminando asimetrías para alcanzar un equilibrio entre las partes y, desde allí, construir un camino cooperativo de unidad y la Alianza del Pacífico responde al modelo de regionalismo abierto, basado en la cooperación para el acceso a los mercados de Asia-Pacífico.

Por otro lado, para entender los vestigios del neoliberalismo en el regionalismo abierto de la Alianza del Pacífico, es necesario precisar qué es Neoliberalismo y cómo llegó a América Latina, para Harvey, es una teoría de prácticas político-económicas, desde la que se afirma que:

La mejor manera de promover el bienestar del ser humano consiste en no restringir el libre desarrollo de las capacidades y de las libertades empresariales del individuo dentro de un marco institucional caracterizado por derechos de propiedad privada fuertes, mercados libres y libertad de comercio. El papel del Estado es crear y preservar el marco institucional apropiado para el desarrollo de estas prácticas (Harvey, 2007, p. 6).
Esta ideología, o doctrina económica, nace con las posturas teóricas de Von Mises y Hayek, en 1947, y se fortalece en el seno de la Universidad de Chicago, donde Hayek enseñó entre 1950 y 1962. Para Hayek (1981) el descubrimiento de la propiedad privada y el mercado fueron la base del desarrollo de la humanidad, el carácter individual y racional del hombre permitió que la humanidad evolucionara de un sistema de vida improductivo, precario y primitivo, a un mundo civilizado productivo y desarrollado.

En esa misma línea Von Mises (1982) establece que los individuos desde su racionalidad están dispuestos a actuar conjuntamente, para alcanzar sus objetivos, por lo que el papel asignado al Estado, es el de salvaguardar la propiedad privada, la libertad y convivencia pacífica.

En atributo, el neoliberalismo, según Gaviria (2004) es un enfoque procedimentalista del bienestar; a partir del cual:

\section{se confía en que la dinámica generada por el mercado conduce de manera automática a la eficiencia y a la equidad, por lo tanto, la libertad económica, es el inicio de la cadena y se expresa en el libre mercado, en el cual de forma natural los precios y los salarios son flexibles, por lo que cualquier desequilibrio en los mercados de bienes y servicios y de trabajo se corregirá de manera automática, mediante la fluctuación de precios y salarios (p.12).}

Esta ideología económica llega 
América Latina con fuerza a través del consenso de Washington en 1990 término acuñado por el economista inglés John Williamson según Lorusso (2015).

Para Davidson (2003), el Consenso surgió de la conferencia "Latin American Adjustment: ¿how much has happened?". La conferencia identificó diez aspectos que sirvieron de base para definir las reformas de política económica que los países deudores debían tener como objetivo. Dichos aspectos son: Disciplina fiscal, recortes al gasto público, reforma tributaria (incluidos los impuestos indirectos y la ampliación de la base tributaria), liberalización financiera, un tipo de cambio competitivo, liberalización del comercio, inversión extranjera directa, privatización de las empresas estatales, desregulación y la protección a los derechos de la propiedad.

En opinión de Dos Santos (2007), el factor determinante para la adopción, a ultranza, de las medidas neoliberales de ajustes estructurales en América Latina, fue la crisis de la deuda de los 80, generada, como es sabido, desde los procesos de industrialización por sustitución de importaciones, el fortalecimiento del mercado doméstico de los 60 y 70 y la modernización tecnológica de los 80 .

Estas medidas estructurales, de corte neoliberal, generaron crecimiento de la desigualdad social en América Latina en la década de los 80,90 y la primera década del siglo XXI, según Millones (2013):

Desde la aplicación de las políticas neoliberales, la pobreza a nivel general muestra una disminución que pasó de un $40.5 \%$ en 1980 a un $32.1 \%$ en 2010. En contraste el ingreso captado por los cuatro deciles más pobres es en promedio menos del $15 \%$ del ingreso total, mientras que el decil más rico capta alrededor de un tercio del mismo (p.56).

En ese período, las caídas en los salarios mínimos reales fueron de mayor magnitud que las de las remuneraciones medias, lo que indica que la incidencia negativa fue mayor para los grupos menos favorecidos.

Para un índice promedio 100 en 1980
la evolución del salario mínimo real
urbano muestra, en los 90, caídas del
$13 \%$ en Chile, $29 \%$ en Uruguay,
$41,5 \%$ en Venezuela, $46 \%$ en Brasil,
$56 \%$ en México, $62 \%$ en Ecuador, y
$76 \%$ en Perú (Alburquerque, 1992,
p.62).

El aumento del desempleo, el empleo de mala calidad, el subempleo generado por las políticas de flexibilización laboral, el fin de los subsidios para productos de consumo, la desaparición de los programas de protección social para las clases medias y populares fueron los generadores de las diferentes formas de inestabilidad social en la región. Respecto a lo cual, Portes y Roberts (2004) plantean que:

Cuando no hay puestos de trabajo disponibles o cuando los que hay son de tan mala calidad que mantienen en la pobreza permanente a quienes los ocupan, los trabajadores formales o los nuevos miembros de la fuerza 
laboral pueden recurrir a formas alternativas a la miseria. Es probable que surjan actividades económicas informales, pero también pueden aparecer otras formas menos convencionales de enfrentar la ausencia de oportunidades de trabajo, como actividades delictivas de diversa índole, incluyendo el narcotráfico, los atracos (p. 79).

Por otro lado, para comprender los vestigios del Socialismo del siglo XXI en el regionalismo cerrado de la CAN, CELAD, ALBA y MERCOSUR hay que precisar que es el Socialismo del XXI. Para Hamburger (2014) el socialismo del Siglo XXI, es una ideología política y económica que:

busca dar respuestas a los graves problemas de subdesarrollo en que vive sumida América Latina, debido fundamentalmente, a los desequilibrios sociales, la injusticia y la inequidad que ha sembrado el modo de producción y las prácticas políticas instauradas, desarrolladas y perpetuadas por el capitalismo (p.135).

Según Harnecker (2011) el término fue acuñado por Hugo Chávez para

diferenciarlo de los errores y desviaciones del llamado "socialismo real" del siglo XX en la Unión Soviética y los países del Este europeo. La lección principal del proyecto chavista es la necesidad e importancia de combinar el socialismo con la democracia, no una democracia liberal, sino una democracia participativa y directa basada en la ética del: amor, la solidaridad, la igualdad entre los hombres, las mujeres entre todos (p.153).

Para Peña y Lillo (2016) el Socialismo del siglo XXI entra de manera fuerte en América Latina por: Venezuela con Hugo Chávez, Bolivia con Evo Morales y por Ecuador con Rafael Correa, los que en los procesos constituyentes introdujeron en sus constituciones la ruta hacia al Socialismo del Siglo XXI. En el caso de otros países de la región como son: Brasil, Argentina, Uruguay, y Paraguay, estos también se sumaron a esta ideología, pero en una versión mucho más moderada. En este mismo contexto, Serrano (2015) plantea que en general el objetivo del Socialismo del siglo XXI se centró en recuperar el rol del Estado, revalorizando su potencial como organizador de la vida política, sacándolo del relego al que lo sometió el neoliberalismo.

\section{Metodología}

Para el abordaje de esta investigación, se utilizó un enfoque cualitativo de orden descriptivo. Un estudio descriptivo "busca especificar propiedades, características y rasgos importantes de cualquier fenómeno que se analice y describe tendencias de un grupo o población" (Hernández, Fernández, y Baptista, 2014, pág.80). Por lo que en este documento se busca describir las características de las iniciativas de integración CAN, MERCOSUR, CELAC, ALBA y la Alianza del Pacífico, las cuales son algunas de las inactivas con mayor protagonismo en América Latina, con el 
fin de identificar si los componentes ideológicos establecidos en los tratados de constitución han limitado el alcance de objetivos sociales y económicos en los países de América Latina. Para resolver estas inquietudes se analizaron, de manera comparada, los acuerdos o tratados de constitución de cada una de ellos y los diversos documentos generados en las diferentes cumbres.

\section{Resultados}

\section{MERCOSUR - Alianza del Pacífico}

El Mercado Común del Sur es una iniciativa de integración comercial y regional constituida a través del tratado de Asunción en 1991 por Argentina, Brasil, Paraguay y Uruguay, a esta iniciativa se incorporaron, en fases posteriores, Venezuela y Bolivia, ésta última en proceso de adhesión. Mediante el Tratado Constitutivo de Asunción, los gobiernos miembros establecieron que:

al 31 de diciembre de 1994 debía estar creado el Mercado Común del Sur (MERCOSUR), lo cual implica libre circulación de bienes, servicios y factores productivos entre los países, a través de la eliminación de los derechos aduaneros y restricciones no arancelarias para la circulación de mercancías, la introducción de un arancel externo común, y la coordinación de políticas macroeconómicas, fiscales y monetarias (MERCOSUR, 1991, Artículos 2 y 3).

En contraste la Alianza del Pacífico, es una iniciativa de integración regional conformada por Chile, Colombia,
México y Perú, creada el 28 de abril de 2011, la cual establece, en el Acuerdo Marco de Constitución, los objetivos de:
a. Construir de manera participativa y consensuada, un área de integración profunda para avanzar progresivamente hacia la libre circulación de bienes, servicios, capitales, personas y economía. b. Impulsar un mayor crecimiento, desarrollo económico y competitividad de las economías de sus integrantes, con miras a lograr mayor bienestar, superar la desigualdad socioeconómica e impulsar la inclusión social de sus habitantes. c. Convertirse en una plataforma de articulación política, integración económica y comercial, y proyección al mundo, con énfasis en la región Asia-Pacífico (Alianza del Pacífico, 2011, p.4).

En principio, se identifican similitudes y diferencias en las dos iniciativas de integración latinoamericanas, descubriendo que se asemejan en el carácter comercial, poseen objetivos compartidos de alcanzar libre circulación de bienes, servicios y capitales o factores productivos entre sus miembros, como mecanismo para alcanzar crecimiento, desarrollo económico y competitividad, por otro lado, en lo social, ambas iniciativas centran en el comercio y el mercado, las bases para lograr mayor bienestar social y reducción de la desigualdad en la población.

En cuanto a las diferencias, el MERCOSUR, es una iniciativa de integración regional hacia dentro, 
mientras que la Alianza del Pacífico es una iniciativa de integración regional hacia afuera, o abierta, la primera pretende potenciar un mercado regional a través de la creación de un mercado común, materializado en la eliminación de los aranceles al interior del bloque, protegiéndose de los intereses comerciales de los gobiernos, de su exterior cercano y lejano, que no hagan parte del mercado común a través de aranceles externos. La segunda, crea zonas de libre circulación de bienes, servicios y capitales, para fortalecer su competitividad y la inserción al mercado del Asia Pacífico, es decir, su principal interés está en los mercados de su exterior lejano.

Por otro lado, el MERCOSUR es la quinta economía del mundo, cuenta con una extensión de $14.869 .775 \mathrm{~km} 2$ posee una de las mayores biodiversidades del mundo, tiene también, el Acuífero Guaraní, una de las reservas de agua dulce más importantes del mundo, así como inmensos recursos energéticos, tanto renovables como no renovable (MERCOSUR, 2020).

La Alianza del Pacífico, por su parte, constituye la octava potencia económica y la octava potencia exportadora a nivel mundial, en 2018 atrajo el 38\% de la inversión extranjera directa de América Latina y el Caribe, en 2019 alcanzó un PIB per cápita de US\$ 19.050 (PPP) y una población de 230 millones de habitantes (Alianza del Pacífico, 2020).

Ambas iniciativas muestran una fuerte tendencia de integración intergubernamental, donde el papel de los gobiernos, a través de los presidentes, los ministros de economía y de comercio exterior en las decisiones son determinantes. En consecuencia en el MERCOSUR el protocolo complementario de Ouro Preto, establece al Consejo del Mercado Común (CMC) como el órgano superior del MERCOSUR, al cual le incumbe la conducción política del proceso de integración y toma de decisiones para asegurar el cumplimento de los objetivos establecidos en el Tratado de Asunción, mientras que al Grupo del Mercado Común (GMC) y la Comisión del Comercio del MERCOSUR (CCM) le otorga capacidad decisoria de naturaleza intergubernamental, y establece como órganos complementarios a la Comisión Parlamentaria Conjunta (CPC), el Foro Consultivo Económico y Social (FCES) y la Secretaria Administrativa del MERCOSUR (SAM) todos con carácter intergubernamental (MERCOSUR, 1994, Art. 2 y 3 ).

Mientras que, en la Alianza del Pacífico, el Acuerdo Marco establece al Consejo de Ministros como el órgano encargado de adoptar decisiones que desarrollen los objetivos y acciones específicas del Acuerdo, no obstante, la máxima instancia en la Alianza del Pacífico es la Cumbre Presidencial (Alianza del Pacífico, 2011). Esto indica el carácter intergubernamental de estas dos iniciativas de integración, distante en el tiempo, una de la otra. Ambas iniciativas muestran una fuerte dinámica en cuanto a cumbres intergubernamentales ejecutadas al interior de cada iniciativa, con el objetivo de fortalecer las 
relaciones y el alcance de los acuerdos y objetivos planteados.

En conclusión, ambas iniciativas centran su atención en lo comercial y económico, una con un fuerte sentir en potenciar el mercado regional, y la otra, con una clara mirada de inserción al libre mercado y a la globalización económica, para lo cual, requieren poner la política al servicio de los objetivos comerciales de cada iniciativa, viabilizando el alcance de los objetivos planteados.

\section{CAN - Alianza del Pacífico}

La Comunidad Andina tiene sus orígenes en 1969, con el Acuerdo de Cartagena, firmado por Bolivia, Colombia, Chile, Ecuador y Perú (Pacto Andino) con el propósito de establecer una unión aduanera en un plazo de diez años, que permitiera mejorar el nivel de vida de sus habitantes, mediante la integración y la cooperación económica y social, sin embargo, este pacto inició con un fuerte carácter proteccionista de integración regional hacia dentro, el cual buscó desarrollar el modelo de industrialización por sustitución de importaciones de los países miembros (Comunidad Andina, 1969). N

\section{$\mathrm{o}$}

obstante, con el transcurrir del tiempo, esta iniciativa flexibiliza su carácter y se transforma en una integración regional abierta, que se materializó en 1997 con el protocolo de Sucre (Comunidad Andina, 1997), el cual persigue los mismos objetivos del Acuerdo de Cartagena de: Promover el desarrollo equilibrado y armónico de los Países Miembros en condiciones de equidad, mediante la integración y la cooperación económica y social; acelerar su crecimiento y la generación de ocupación; facilitar su participación en el proceso de integración regional, con miras a la formación gradual de un mercado común latinoamericano.

\begin{abstract}
Asimismo, son objetivos de este Acuerdo propender por disminuir la vulnerabilidad externa y mejorar la posición de los Países Miembros, en el contexto económico internacional; fortalecer la solidaridad subregional y reducir las diferencias de desarrollo existentes entre los Países Miembros. Estos objetivos tienen la finalidad de procurar un mejoramiento persistente en el nivel de vida de los habitantes de la Subregión (Comunidad Andina, 1969, Art. 1).
\end{abstract}

Pero bajo un esquema de integración regional abierta, para lo cual se establecen mecanismos que permitan alcanzar los objetivos, además el Protocolo de Sucre (Comunidad Andina, 1997) otorga, al Consejo de Ministros de Relaciones Exteriores, la facultad de establecer política exterior común, para los asuntos de interés subregional, y concertar posición política conjunta que permita a la región participar de manera colegiada en foros y organizaciones políticas internacionales, es decir, el cambio del modelo de integración a todas luces da un giro, del regionalismo cerrado al regionalismo abierto, pero manteniendo un fuerte componente de integración intergubernamental, dado que las decisiones presidenciales y de los ministros son las encargadas de materializar cada objetivo, sin 
desconocer la participación que esta iniciativa le da a la población civil a través del Consejo Consultivo de Empresarios, el Consejo Consultivo Laboral, Consejo Consultivo de Pueblos Indígenas entre otros, pero sin carácter vinculantes de sus decisiones.

En contraste, la estrategia de integración de la Alianza del Pacífico en vez de buscar un mercado común regional, busca establecer una zona de libre comercio para la inserción al Asia Pacífico, donde la libre circulación de mercancía y de capital tiene la mayor relevancia. En cuanto a la política exterior de la Alianza del Pacífico se enfoca a establecer mecanismos conjuntos para generar condiciones a la inversión extranjera directa bajo el esquema del libre mercado, mientras que en los asuntos de interés regional desde lo político si no afectan el interés comercial y económico de los miembros de la Alianza, cada uno de manera separada podrá establecer su posición la cual no afectará el comportamiento de los objetivos de la integración, contrario a la posición colegiada de política exterior que busca establecer la CAN.

Por otro lado, estas dos iniciativas, en principio, se asemejan en el fuerte carácter intergubernamental de la integración, dado que en ambas el papel de los gobiernos es preponderante en la articulación y establecimiento de protocolos y declaraciones conjuntas, donde el papel de la sociedad civil es consultivo, no vinculante para las decisiones finales. La CAN cuenta con el $20 \%$ de la biodiversidad del planeta, el
$10 \%$ del agua dulce del mundo, el $35 \%$ de los bosques de América Latina y el Caribe, un territorio de $3.798 .000 \mathrm{~km} 2$ (Comunidad Andina, 2011).

En conclusión, la CAN es una iniciativa de integración comercial que busca fortalecer el mercado regional, (regionalismo cerrado), como estrategia de lucha contra los riesgos externos derivados de la globalización, la Alianza del Pacífico en contraste también es una iniciativa comercial pero que mira más al mercado global, que al mercado regional, (regionalismo abierto) como estrategias de posicionamiento global que le genere incidencia en un mercado ampliado, ambas iniciativas ponen a merced del mercado los problemas sociales como la pobreza y la desigualdad que enfrenta la región.

\section{CELAC - Alianza del Pacífico}

La Comunidad de Estados Latinoamericanos y caribeños (CELAC) es un mecanismo intergubernamental que busca priorizar el diálogo y concertación política. Integrado por los treinta y tres países de América Latina y el Caribe. Materializado en la cumbre de Caracas en 2011, el propósito del mecanismo es avanzar, gradualmente, en el proceso de integración de la región, haciendo un sabio equilibrio entre la unidad y la diversidad política, económica, social y cultural de los habitantes de América Latina y el Caribe. La CELAC desde su creación aspira a ser una voz única en decisiones políticas estructuradas en el ámbito político y la cooperación en apoyo de los programas de integración 
regional, sustituyendo al grupo de Rio. La CELAC tiene como objetivos: constituir un gran bloque de fuerza política, económica y social que les identifique dentro y fuera de la región. Establecer programas que permitan avanzar hacia la construcción de un mundo multipolar y en paz. Avanzar en la integración en diversas áreas, bajo los principios de respeto a la democracia, las riquezas naturales, la sostenibilidad y la soberanía de todos los países miembros. Además, establece como principio rector el respeto al Derecho Internacional, la solución pacífica de controversias, la prohibición del uso y de la amenaza del uso de la fuerza, el respeto a la autodeterminación, el respeto a la soberanía, el respeto a la integridad territorial, la no injerencia en los asuntos internos de cada país, la protección y promoción de todos los derechos humanos y de la democracia como valores y principios fundamentales para abordar los procesos de diálogo, intercambio y negociación política que se activen desde dentro de esta iniciativa (CELAC, 2011).

En conclusión, la iniciativa de integración Alianza del Pacífico representa una integración comercial y económica mientras que la CELAC representa una iniciativa de integración política que aborda desafíos económicos y comerciales, es decir, en principio estas dos iniciativas son de naturaleza opuestas, pero no quiere decir que no puedan converger en objetivos comunes, en lo concerniente a la reducción de la pobreza, disparidades sociales y el mantenimiento de los principios democráticos de la región.

\section{ALBA - Alianza del Pacífico}

La Alianza Bolivariana para los Pueblos de Nuestra América - Tratado de Comercio de los Pueblos (ALBA-TCP) es una iniciativa de integración de los Estados de Venezuela, Cuba, Bolivia, Nicaragua, Mancomunidad de Dominica, Antigua y Barbuda, Ecuador, San Vicente y Las Granadinas, Santa Lucía, San Cristóbal y Nieves y Granada, la cual es considerada por sus fundadores como:

\begin{abstract}
una plataforma de integración política e ideológica de los países de América Latina y el Caribe, que pone énfasis en la solidaridad, la complementariedad, la justicia y la cooperación, tiene el propósito histórico fundamental de unir las capacidades y fortalezas de los países que la integran, en la perspectiva de producir las transformaciones estructurales y el sistema de relaciones necesarias para alcanzar el desarrollo integral requerido para la continuidad de la existencia como naciones soberanas y justas. Es, además, una alianza política, económica y social en defensa de la independencia, la autodeterminación y la identidad de los pueblos que la integran (ALBA TCP, 2016).
\end{abstract}

Los principios fundamentales del ALBA son: el comercio y la inversión no deben ser fines en sí mismos, debe existir un trato especial y diferenciado a los países, la complementariedad económica y la cooperación entre los países participantes, y la no competencia entre países y producciones, cooperación $\mathrm{y}$ 
solidaridad que se exprese en planes especiales para los países menos desarrollados en la región, creación del Fondo de Emergencia Social, desarrollo integrador de las comunicaciones y el transporte entre los países latinoamericanos y caribeños, acciones para propiciar la sostenibilidad del desarrollo mediante normas que protejan el medio ambiente, integración energética entre los países de la región, fomento de las inversiones de capitales latinoamericanos en la propia América Latina y el Caribe, defensa de la cultura latinoamericana y caribeña y de la identidad de los pueblos de la región, medidas para las normas de propiedad intelectual, concertación de posiciones en la esfera multilateral y en los procesos de negociación de todo tipo con países y bloques de otras regiones (ALBA TCP, 2016).

El ALBA se puede decir que se constituyó en una iniciativa de regionalismo multidimensional, principalmente política, que centra su accionar en la lucha contra la pobreza y el desarrollo social de los pueblos de América, alejada de criterios mercantilistas que socavan los principios de solidaridad, cooperación y complementariedad productiva de los pueblos. Para lo cual cuenta con el consejo presidencial, el cual es un órgano integrado por los Jefes de Estado y/o de Gobierno y la máxima instancia de deliberación, decisión y orientación política de la Alianza. Sin embargo, la participación de la población civil en los diferentes consejos es permanente y decisiva en que en las decisiones adoptadas.

En contraste la Alianza del Pacífico representa una iniciativa de integración donde el principal objetivo es la creación de un área de integración profunda para alcanzar una zona de libre circulación de bienes, servicios, capitales y personas. Opuesto al planteamiento del ALBA donde el comercio y la inversión no son un fin en sí mismo, sino un instrumento para alcanzar un desarrollo sostenible, dado que no se considera la ampliación del mercado como el mecanismo idóneo de integración, sino la cooperación y complementariedad productiva de los Estados miembros.

Por otro lado, la Alianza del Pacífico es una iniciativa de integración económica, mientras que el ALBA es una integración política que busca resolver a través de la cooperación y solidaridad de los pueblos las disparidades económicas en los países.

En conclusión, las diferentes iniciativas de integración presentadas en América Latina presentan un fuerte componente de multipluralismo asociativos y multipluralismo participativo. Así también, se observan diferencias profundas en el modo de abordar cada iniciativa de integración con respecto al propósito de la Alianza del Pacífico.

Las iniciativas de integración MERCOSUR y CAN, CELAC, ALBA en principio buscan a través del regionalismo cerrado potenciar el mercado intrarregional para resolver problemas de pobreza, disparidades sociales y económicas en la región, entendiendo que se debe aunar esfuerzos para proteger el continente de fuertes 
efectos del libre mercado, para lo cual la participación política en cada iniciativa es fundamental, mientras que en la Alianza del Pacífico se sobrepone los intereses económicos por encima de los políticos y sociales, abrazando así un regionalismo abierto donde la integración profunda, el neoliberalismo y el libre mercado son la línea de ruta para resolver los problemas económicos y sociales de las países asociados.

En este orden, el Acuerdo Marco que da origen a la Alianza del Pacífico inicia con una retórica de integración economicista, con evidente tinte neoliberal, donde el mercado es el mejor mecanismo para reducir los grandes desequilibrios sociales que enfrentan los países que la integran y lo establece de la siguiente manera:

Convencida que la integración económica regional constituye uno de los instrumentos esenciales para que los Estados de América Latina avancen en su desarrollo económico y social sostenible, promoviendo una mejor calidad de vida para sus pueblos y contribuyendo a resolver los problemas que aun afectan a la región, como la pobreza, la exclusión, y la desigualdad social persistente (Alianza del Pacífico,2011, p.2 ).

El Artículo 3 del Acuerdo Marco de Constitución de la Alianza del Pacífico establece los siguientes objetivos:

a. Construir, de manera participativa y consensuada, un área de integración profunda para avanzar progresivamente hacia la libre circulación de bienes, servicios, capitales y personas; $b$. Impulsar mayor crecimiento, desarrollo y competitividad de las economías de las partes, con miras a lograr un mayor bienestar, la superación de la desigualdad socioeconómica y la inclusión social de sus habitantes; y c. Convertirse en una plataforma de articulación política, de integración económica y comercial, y de proyección al mundo, con especial énfasis al Asia Pacífico (Alianza del Pacífico, 2011).

Estos objetivos sin duda dejan al mercado y la libre circulación de mercancía, de capital y de personas, el papel principal en la lucha contra la desigualdad y la exclusión social de la población, paradójicamente los factores que han profundizado estos males en América Latina. En coherencia a las acciones establecidas en el artículo 3 para alcanzar los objetivos, se alinean las postura neoliberal y economicista de los problemas sociales que enfrenta la región, donde el actuar eficiente del mercado permitirá reducir de manera drástica la desigualdad y la exclusión social de la población.

Estas acciones son: a. Liberalizar el intercambio comercial de bienes y servicios, con miras a consolidar una zona de libre comercio entre las partes, a. Avanzar hacia la libre circulación de capitales y la promoción de la inversión entre las partes, c. desarrollar acciones de facilitación del comercio y asuntos aduaneros; d. Promover la cooperación entre las autoridades migratoria $y$ consulares y facilitar el movimiento de personas y de transito migratorio en el 
territorio de las partes; e. Coordinar las prevención y contención de la delincuencia organizada y transnacional para fortalecer las instancias de seguridad pública y de procuración de justicia de las partes; y f. Contribuir a la integración de las partes mediante el desarrollo de mecanismos de cooperación e impulsar la plataforma de cooperación del Pacífico suscrita en diciembre de 2011 en las áreas definidas (Alianza del Pacífico, 2011).

Como se puede observar ningunas de las acciones propuestas en el Acuerdo Marco para alcanzar los objetivos apunta a mejorar el bienestar, la superación de la desigualdad socioeconómica y la exclusión social de los habitantes de los países integrados, todas apuntan a fortalecer el libre mercado y el crecimiento económico, situación que profundiza los males sociales propuestos a combatir con esta Alianza.

Las acciones propuestas ya han sido utilizadas en algunos países con modelo económico similar donde la desregulación del mercado es el arma de lucha contra los problemas sociales, pero con resultados sociales devastadores (Stiglitz, 2012). No obstante, la lucha contra la endémica desigualdad y la exclusión social en los países que conforman la Alianza del Pacífico no se puede dejar solo en manos del mercado, dado que la experiencia demuestra la ineficacia del mercado en la lucha contra ese mal social. La fuerza del mercado, crea de manera natural la desigualdad, sin embargo, para Stiglitz (2012) la desigualdad moderna es una consecuencia, tanto de las fuerzas políticas como económicas, por lo tanto, los gobiernos tienen la posibilidad de trasladar dineros de la parte superior a la inferior y a la intermedia, o viceversa, a través de los impuestos, y los subsidios de esta manera puede alterar las dinámicas de las riquezas.

Aun así, las acciones propuestas en el Acuerdo Marco parecieran que van en contravía de mejorar el bienestar de la población menos favorecida y excluida naturalmente por el mercado, debido a que su énfasis está en la desregulación de los mercados, la libre circulación de mercancía y de capitales, profundizando así la exclusión a través de la poca productividad marginal de la población, que no tienen acceso al sistema educativo de calidad y seguridad social. En consecuencia, la contraposición de fuerzas entre las iniciativas, antes mencionadas, de regionalismos cerrados de la CAN, MERCOSUR, CELAC Y ALBA, en alcanzar los objetivos sociales a través de priorizar las relaciones intrarregionales, instancia donde la cooperación en asuntos estratégicos desde lo político, económico, ambiental y cultural se opone a la postura de la Alianza del Pacífico, en la cual el libre mercado es el mecanismo idóneo para solucionar los problemas sociales y económicos, de acuerdo a lo planteado en los objetivos del Acuerdo Marco.

Consciente de todo lo descrito, y al analizar la Alianza del Pacífico a través del Acuerdo Marco, se evidencia un fuerte vestigio neoliberal, al pretender que el mercado será el mecanismo adecuado para la reducción de los 
desequilibrios sociales que enfrentan los países asociados, al respecto Mejía (2018), establece que:

\begin{abstract}
la Alianza del Pacífico es un mecanismo de integración que no tiene incidencia positiva en los indicadores sociales de pobreza, desigualdad y exclusión social en los países que la integran, pero que si repercute de manera significativa en generar grandes beneficios económicos y comerciales para algunos pocos (p.229).
\end{abstract}

Resultados iguales a los generados por las políticas de corte neoliberal presentes históricamente en América Latina.

Por lo tanto, las acciones propuestas en el Acuerdo Marco como: Liberalizar el intercambio comercial de bienes y servicios, con miras a consolidar una zona de libre comercio entre las partes; avanzar hacia la libre circulación de capitales y la promoción de la inversión entre las partes; desarrollar acciones de facilitación del comercio $y$ asuntos aduaneros, son el rastro de las medidas de corte neoliberal propuesta e implementadas en América Latina. Estas medidas tienen un fuerte interés en potenciar el mercado, de tal manera que la acción individual de las personas pueda llevarlos a distribuir, de manera eficiente, los recursos, llevando a resolver, de manera automática, los problemas sociales asociados a la pobreza, desempleo, desigualdad y exclusión social que enfrentan los países integrados.

En base en lo anterior, se puede evidenciar la contraposición de fuerzas de esta iniciativa de integración la Alianza del Pacífico, basada en el regionalismo abierto y las iniciativas asociadas al regionalismo cerrado heredadas de la CAN, MERCOSUR, CELAC y ALBA, las cuales buscan resolver los problemas sociales de América Latina a través de la cooperación entre los Estados, donde la protección regional del mercado y la ampliación de la capacidad política para reaccionar, de manera conjunta, ante hechos que afecten los intereses regionales en diferentes ámbitos son la verdadera línea de ruta.

\section{Conclusiones}

Las iniciativas de integración en América Latina, presentan de manera divergente mecanismos para alcanzar sus objetivos, algunas como la CAN, MERCOSUR y la Alianza del Pacífico ponen un gran interés en el mercado para solucionar problemas sociales, mientras que iniciativas como CELAC Y ALBA, ponen el interés en la cooperación política y económica de los Estados como mecanismo idóneo para lograr superar los crónicos problemas sociales que enfrenta la región.

Existe una fuerte lucha de modelos económicos entre los países de América Latina, lo cual no ha permitido lograr una visión conjunta de región, dificultando así el tránsito a una sociedad más justa y menos desigual que supere las graves problemáticas sociales que la aquejan, debido a que es imposible que, de manera individual, cada país pueda transformar 
los estructurales desequilibrios sociales y económicos que enfrenta.

En cada una de las iniciativas estudiadas se observan elementos ideológicos del Socialismo del Siglo XXI o del Neoliberalismo, que dificulta establecer puntos de convergencia que permita concurrir al alcance de objetivos social y económico conjuntos, lo cual debilita la capacidad de la región para mostrarse al mundo como un bloque sólido que busque ser un actor determinante en la política y economía mundial.

En consecuencia, se deben superar las vanidades y egos gubernamentales para poder avanzar en la construcción de una América Latina, próspera, incluyente e influyente, debido a que, en las múltiples iniciativas de integración firmadas, existe una exagerada retórica que refleja los intereses o voluntad de algunos de los Estados, pero que no se articula con políticas públicas tendientes a mejorar la calidad de vida de sus habitantes.

Se debe prestar mucha atención para no repetir los errores del pasado. Cuando se le entregó a ciegas al mercado la labor de resolver los problemas sociales de la región, y que al final mostró resultados nefastos, pero esto no quiere decir que se deben tomar decisiones de extremos en la integración de América Latina, lo que se debe priorizar es la concertación donde las necesidades superiores de la sociedad se resuelvan a través de la cooperación, sin pretensión de dominación y de pérdida de soberanía de los Estados ante imposiciones de otros.
América Latina debe brillar con luz propia, por lo tanto, hay que superar la agotada y lapidaria discusión, entre izquierda y derecha, hay que avanzar en la construcción de agenda de concertación multimodal, geoestratégico y pragmática en el ámbito político y económico, en el seno de las iniciativas de integración para poder establecer líneas de ruta reales que permitan resolver los apremiantes problemas sociales de la región. Como lo plantean Mejía y Vargas (2018) y Guadarrama (2020).

La evidente lucha ideológica entre modelos de integración no permite avanzar en la consolidación de una América Latina influyente en la esfera global, es necesario avanzar a una convergencia de modelo, donde se depongan los intereses particulares de cada Estado y se prioricen los intereses generales de la región, de otra manera, las iniciativas de integración seguirán siendo más retóricas que prácticas. Debido a que, tanto el Regionalismo Abierto, materializado en la Alianza del Pacífico, poseedor de vestigios Neoliberales no ha sido una alternativa eficiente en lograr mayor bienestar social y efectiva integración de los pueblos, como tampoco el regionalismo cerrado, evidente en las demás iniciativas de integración vigentes en América Latina.

\section{Referencias}

ALBA-TCP. (2020). ¿Qué es el ALBA-TCP? Recuperado de http://albatcp.cubaminrex. $\mathrm{cu} /$ page/alba-tcp

Alburquerque, F. (1992). La crítica situación de 
América Latina al inicio de los noventa y la necesidad de una estrategia de desarrollo alternativa al neoliberalismo. América Latina Hoy, (4), 57- 68.

Alianza del Pacífico. (2011). Acuerdo Marco de la Alianza del Pacífico. Recuperado de https://alianzapacifico.net/download/acue rdo-marco-de-la-alianza-del-pacifico/

Alianza del Pacífico. (2020). Cifras Alianza del Pacífico el poder de la Integración. Recuperado de https://alianzapacifico.net/

Aranda, G., y Salinas, S. (2015). ALBA y Alianza del Pacífico: ¿Choque de integraciones? UNIVERSUM, 30(1), 17-38.

Botero, J. (2013). Una integración dividida: las múltiples iniciativas que generan dificultades para lograr una integración regional en Latinoamérica. Punto de vista, 4(7), 123-132.

Botto, M. (2015). América del Sur y la integración regional: ¿Quo vadis? Los alcances de la cooperación regional en el MERCOSUR. CONfines de relaciones internacionales $y$ ciencia política, 11(21), 9-38.

Bull, H. (1977). The Anarchical Society. A study or order in world politics. Londres: Macmillan.

Caballero, S. (2013). Integración en América Latina: lógicas en pugna. Politica Exterior, 27(154), 136-141.

CELAC. (2011). Cumbre CELAC Caracas, Venezuela 2011. Recuperado de http://www.sela. org/celac/cumbres/

Comunidad Andina. (1969). Acuerdo de Cartagena. Recuperado de http://www.comunidad andina.org/StaticFiles/DocOf/DBASICO 1.pdf

Comunidad Andina de Naciones. (1997). Protocolo de Sucre. Recuperado de http://www. comunidadandina.org/Documentos.aspx
Comunidad Andina. (2011). Ya son doce los sitios establecidos en la CAN para monitorear el impacto climático en ecosistemas. Recuperado de http://www.comunidad andina.org/index.aspx

Davidson, P. (2003). ¿Qué es erróneo en el consenso de Washington y qué debemos hacer? Cuadernos de Economía, 22(39), 169-194.

Dos Santos, T. (2007). Del terror a la esperanza. Auge y decadencia del neo-liberalismo. Caracas: Monte Ávila.

Gaviria, M. (2004). Neoliberalismo y Desarrollo en América Latina una discusión sobre las reformas estructurales promovidas por el "Consenso de Washington". Revista Académica e Institucional de la UCPR, (69), 7-21.

Grynspan, R. (2015). Productividad e Integración Comercial en América Latina: Tendencias y Desafíos. ICE: Revista de Economía, (884), 51-66.

Guadarrama, P. (2004). El pensamiento de la integración latinoamericana ante la globalización. Cuadernos Americanos: Nueva época, 1(103), 34-59.

Guadarrama, P. (2019). Papel de la Ilustración latinoamericana en la gestación de la cultura integracionista. Cultura Latinoamericana, 30(2), 118-145. doi:http://dx.doi.org/ 10.14718/CulturaLatinoam.2019.30.2.6

Guadarrama, P. (2020). Nuevas derechas ante la integración. En J.Estrada, C. Jiménez, y J. Puello-Socarrás (Eds), Contra Nuestra América. Estrategias de la derecha en el siglo XXI (pp. 185-205). Buenos Aires: CLACSO.

Haas, E. (1958). The uniting of Europe. Political, social and economical forces, 1950-1957. Stanford: Stanford University Press.

Hamburger, A. (2014). El Socialismo del Siglo 
XXI en América Latina: Características, desarrollos y desafíos. Revista de Relaciones Internacionales, Estrategia y Seguridad, 9(1), 131-154.

Harnecker, M. (2011). Democracia y socialismo: el futuro enraizado en el presente. Estudios críticos del desarrollo, 1(1), 151-182.

Harvey, D. (2007). Breve Historia del Neoliberalismo. Madrid: Editorial AKAL.

Hayek, F. A. (1981). Los Fundamentos Éticos de una Sociedad Libre. Estudios Públicos, (3), $71-82$.

Hernández, S. R., Fernández, C. C., y Baptista, L. P. (2014). Metodología de la Investigación (Sexta edición). México: McGraw Hill.

Lorusso, F. (2015). Management y privatismo: Pilares ideológicos del neoliberalismo y la americanización en América Latina. Politica y cultura, (43), 95-123.

Márquez, L., y Recalde, M. L. (2017). Causas y efectos de la integración económica en América Latina. Actualidad Económica, 27(92), 5-11.

Mejía, M. (2018). Alianza del Pacífico, un Desafío Social a la Integración Comercial. Soft Power, 5n(1), 215 - 244. DOI: 10.17450/ 180112

Mejía, M., y Vargas, A. (2018). La relación: RusiaAmérica Latina, entre el pragmatismo económico y la configuración de un mundo multipolar y balanceado. Observatorio Colombiano de Tratados Comerciales OCTC, (5), 18-21.

MERCOSUR. (1991). Tratado de Asunción. Recuperado de http://www.mercosur.int/

MERCOSUR. (1994). Protocolo de Ouro Preto. Recuperado de https://www.mercosur.int/ documentos-y-normativa/tratados/
MERCOSUR. (2020). En pocas palabras. Recuperado de https://www.mercosur.int/quienes-som os/en-pocas-palabras/

Millones, M. (2013). Neoliberalismo en América Latina: una interpretación desde la ideología en Žižek. Sociológica (México), 28(79), 51-78.

Penneta. P. (2011), Integración e integraciones Europa, América Latina y el Caribe. Bogotá: Planeta.

Peña y Lillo, J. (2016). América Latina y el socialismo del siglo XXI. Revista San Gregorio, (15), 112-121.

Portes, A., y Roberts, B. (2004). Empleo y desigualdad urbanos bajo el libre mercado: Cosecuencias del experimento neoliberal. Nueva Sociedad, (193), 77-96.

Serrano, A. (2015). América Latina en disputa. Caracas: Fundación Editorial El perro y la rana.

Stiglitz, J. E. (2012). El Precio de la Desigualdad. El 1\% de la población tiene lo que el 99\% necesita para vivir. Bogotá: Taurus.

Tremolada, E. (2015). ¿La Alianza del Pacífico facilita la inserción de Colombia en la región Asia -Pacífico? Papel Político, 19(2), 721-752.

Urueña, R. (2008). Derecho de las organizaciones internacionales. Bogotá: Uniandes-Temis.

Vieira, E. (2005). Evolución de las Teorías Sobre Integración en el Contexto de las Teorías de Relaciones Internacionales. Papel Político, (18), 235-290.

Von Mises, L. (1982). Liberalismo. Sao Paulo: Unión Editorial. 